\title{
ANÁLISE DO EFEITO DA INJEÇÃO DE GÁS NO DISTRIBUIDOR PARA A REMOÇÃO DE INCLUSÕES*
}

\author{
Alexandre de Freitas Gomes de Mendonça ${ }^{1}$ \\ Melissa Souza de Faria ${ }^{2}$ \\ Daniel Andrade de Jesus ${ }^{2}$ \\ Gabriel Novaes da Silva ${ }^{2}$ \\ Augusto Luis Almeida Santos ${ }^{2}$ \\ Adrielle Cintra Filgueira ${ }^{2}$ \\ Bernardo Martins Braga ${ }^{3}$ \\ Roberto Parreiras Tavares ${ }^{4}$
}

\section{Resumo}

A remoção de inclusões nos distribuidores de lingotamento contínuo tem sido investigada por meio de modelamentos físicos e matemáticos. Recentemente, a injeção de gás nos distribuidores tem sido considerada como uma alternativa para aprimorar a remoção de inclusões. Vários trabalhos vêm sendo desenvolvidos analisando os efeitos da injeção de gás e sua interação com as inclusões. No entanto, existe espaço para mais investigações envolvendo o efeito da vazão do gás e a localização do sistema de injeção de gás na eficiência de remoção de inclusões. No presente trabalho, foi usado um modelo físico construído em escala 1:3 de um distribuidor industrial de 40 toneladas de capacidade nominal, para analisar o efeito da injeção de gás. Os experimentos incluíram contagem on-line de partículas pela sonda APS III e análise de curvas DTR. $O$ resultado mostrou que a injeção de gás é benéfica à remoção de inclusões e que a análise pura dos volumes característicos, calculados a partir das curvas DTR, não é suficiente para avaliar a eficiência de remoção.

Palavras-chave: Distribuidor; Injeção de gás; Inclusões; Modelamento físico.

\section{ANALYSIS OF EFFECT OF GAS INJECTION IN TUNDISH TO INCLUSION REMOVAL}

\begin{abstract}
Inclusion removal in continuous casting tundishes has been extensively investigated by means of physical and mathematical modeling. More recently, gas injection in tundishes has been considered as an alternative to improve inclusion removal. Several works have been developed analyzing the effect of gas injection and its interactions with inclusions, however, there is room for more investigations regarding the effect of gas flow rate and location of the gas injection system on the inclusion removal efficiency. In the present work, a physical model of an industrial tundish of 40 metric tonnes of nominal capacity, built in a 1:3 scale, was used to analyze the effect of gas injection. The experiments included online particle counts by APS III probe and analysis RTD curves. The results show that the injection of gas is beneficial to the removal of inclusions and the analysis of the characteristic volumes of RTD curves is not sufficient to conclude about removal efficiency.
\end{abstract}

Keywords: Tundish; Gas injection; Inclusion; Physical modeling.

1 Engenheiro Metalurgista, CQE/ASQ, Estudante do PPGEM/UFMG, Belo Horizonte, MG, Brasil.

2 Estudante de Engenharia Metalúrgica da UFMG, Belo Horizonte, Minas Gerais, Brasil.

3 Engenheiro Metalurgista, M. Sc., Estudante do PPGEM/UFMG, Membro da ABM, Belo Horizonte, Minas Gerais, Brasil.

4 Engenheiro Metalurgista, PhD, Professor do Departamento de Engenharia Metalúrgica da UFMG, Membro da ABM, Belo Horizonte, Minas Gerais, Brasil. 


\section{INTRODUÇÃO}

A produção do aço é feita na aciaria em três etapas básicas de processamento: refino primário, refino de panela (metalurgia secundária) e o lingotamento contínuo. As etapas de refino cuidam da composição química, limpidez e ajuste de temperatura do aço líquido para o lingotamento. Esse último processo, por sua vez, é responsável pela solidificação do aço líquido de forma controlada para atender aspectos superficiais e internos de produto.

Após as etapas de refino, é desejável que o aço líquido apresente um nível de inclusões que não comprometa a lingotabilidade, e principalmente, a qualidade interna e superficial do produto final. Inúmeras técnicas são aplicadas para o controle de inclusões nos refinos e no lingotamento. No lingotamento, em particular, o distribuidor pode ser projetado para desempenhar esse papel, além das funções originais de reservatório e distribuição do aço líquido.

Para maximizar a oportunidade de flotação de inclusões em um distribuidor, é conveniente inserir modificadores de fluxo em seu interior, como diques, barragens, inibidores de turbulência, dentre outros. Em geral, esses modificadores são estruturas sólidas que atuam como obstáculos físicos e alteram o perfil de escoamento, agregando ao aço melhor qualidade. Além dos modificadores de fluxo citados, o uso de cortina de gás [1] pode também desempenhar papel similar e potencializar a flotação de inclusões. $\mathrm{Na}$ indústria, a cortina de gás pode ser criada pela colocação de um elemento poroso na base do distribuidor que é alimentado pela injeção de um gás inerte, normalmente argônio. O elemento poroso, ao desprender gás argônio, cria uma série de bolhas de gás que sobem até a superfície do aço líquido no distribuidor. Durante esse percurso de ascensão, as bolhas que colidirem com as partículas de inclusões não metálicas, poderão aderir a elas, conduzindo-as para a escória do distribuidor em um processo similar ao de flotação [2].

Com base nesses fatos, a remoção de inclusões no distribuidor de lingotamento contínuo tem sido intensamente investigada por meio de modelamentos físicos e matemáticos. Recentemente, a injeção de gás no distribuidor tem sido considerada como uma alternativa para aprimorar a remoção de inclusões. Vários trabalhos vêm sendo desenvolvidos analisando os efeitos da injeção de gás e sua interação com as inclusões [3-6]. No entanto, há espaço para mais investigações envolvendo o efeito da vazão do gás, da localização do sistema de injeção de gás e das dimensões das bolhas na eficiência de remoção de inclusões.

O presente trabalho visou elucidar as mudanças geradas do sistema, submetido à injeção de gás, assim como, determinar a melhor configuração do distribuidor para a flotação de inclusões submetidas a três níveis de posicionamento do plugue poroso e a quatro níveis de vazão de gás para cada posição de plugue. A eficiência de remoção de inclusões foi analisada pelo emprego da sonda de contagem de inclusões APS III, Aqueous Particle Sensor 3. As inclusões foram simuladas por microesferas ocas de vidro de borossilicato, de tamanho conhecido e vazão de injeção da polpa de inclusões controlada. A sonda APS III fez a contagem on-line de inclusões na saída do veio. Adicionalmente, a análise dos volumes característicos foi feita através do levantamento de curvas de distribuição de tempos de residência, DTR [7]. Por fim, foram confrontados a eficiência de remoção efetiva com os parâmetros de escoamento obtidos pelas curvas DTR e apontada qual configuração é mais adequada. 


\section{MATERIAIS E MÉTODOS}

\subsection{Modelo físico do distribuidor}

O modelo físico do distribuidor utilizado encontra-se instalado no Laboratório de Simulação de Processos, LaSiP, do Departamento de Engenharia Metalúrgica e de Materiais da Universidade Federal de Minas Gerais. Ele foi construído em acrílico na escala reduzida de 1:3 de um distribuidor real em operação e possui uma válvula de entrada e duas de saída. O modelo físico respeita os aspectos de similaridade [8] geométrica e dinâmica. Desta forma é possível fazer inferências do comportamento do escoamento pela aplicação de técnicas específicas e transportar o resultado para o ambiente industrial, o que elimina a necessidade de fazer testes diretamente na planta. A transposição do modelo físico para o industrial, e vice-versa, foi feita através do critério de similaridade baseado no número de Froude.

A geometria e dimensões do modelo físico do distribuidor que foi usado para o presente estudo podem ser verificadas na Figura 1.
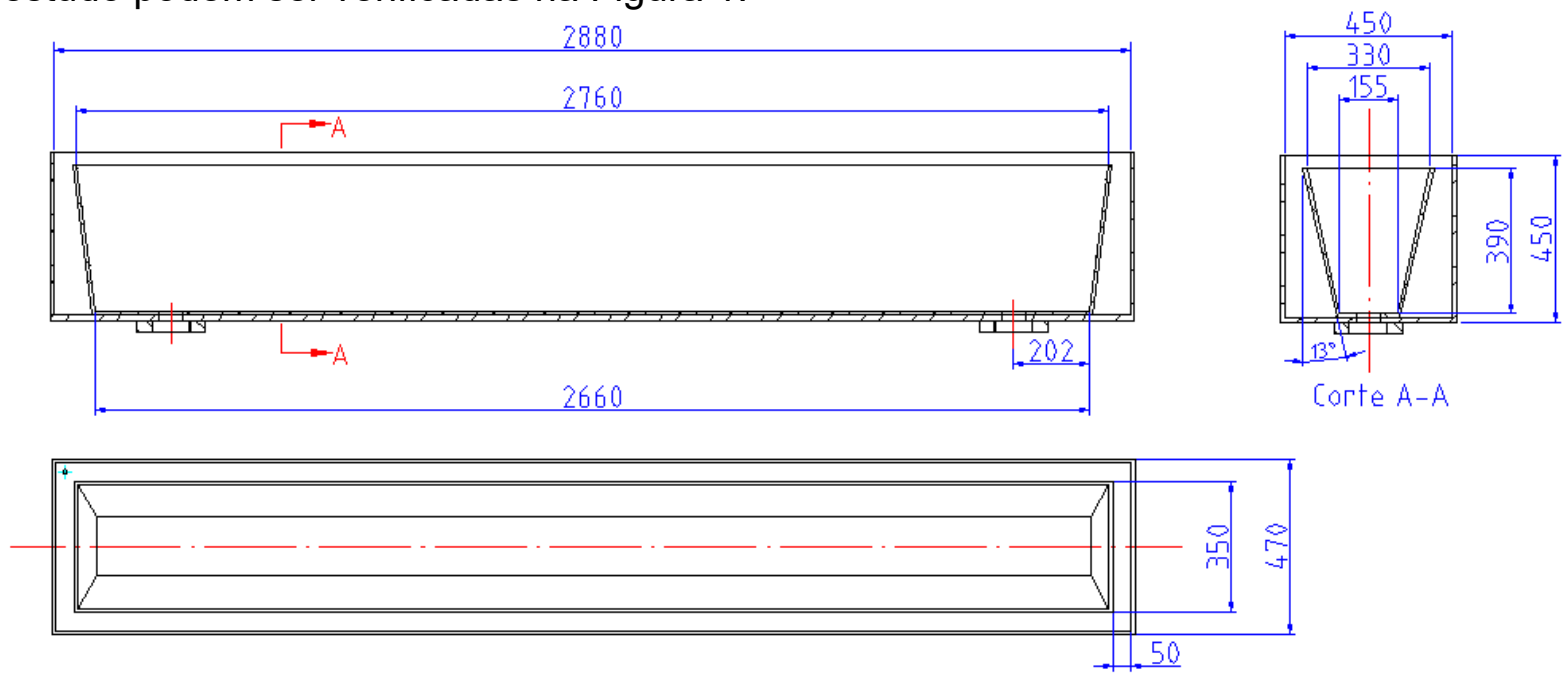

Figura 1. Desenho técnico do modelo físico do distribuidor (dimensões em mm).

A operação do modelo físico é automatizada e integrada por um sistema de cartões de entrada e saída monitorados e controlados pelo programa SoftPLC, que emula o funcionamento de um CLP (Controlador Lógico Programável). Os sistemas de controle e supervisão controlam as seguintes variáveis:

- vazão de alimentação do distribuidor;

- nível de líquido no distribuidor.

\subsection{Condição de operação dos ensaios}

O distribuidor foi preenchido com um volume de 167 litros de água (correspondendo a 31,7 toneladas de aço no distribuidor) atendendo os critérios de similaridade. Depois de estabelecido o nível de operação, os veios foram abertos, permitindo que a soma das vazões de saída fosse a mesma de entrada, mantendo assim uma coluna de água constante no distribuidor. Para a injeção de gás, dois plugues porosos foram instalados na base do distribuidor e equidistantes em relação a válvula longa. Na Figura 2 é ilustrado um plugue usado. Foram testadas três posições de plugue: 28,56 e $84 \mathrm{~cm}$ em relação a entrada do sistema. As vazões de gás injetado foram: 1, 2, 3 e $4 \mathrm{NI} / \mathrm{min}$. Para cada combinação de posição de plugue e vazão de gás estudada, foram feitos testes com repetições. A vazão de água na entrada do distribuidor foi mantida em $50 \mathrm{l} / \mathrm{min}$ (o equivalente a $5,48 \mathrm{t} / \mathrm{min}$ da panela para o distribuidor industrial). A saída em cada veio 
operou com $25 \mathrm{I} / \mathrm{min}$, correspondente a uma vazão mássica de aço de 2,74 t/min por veio.

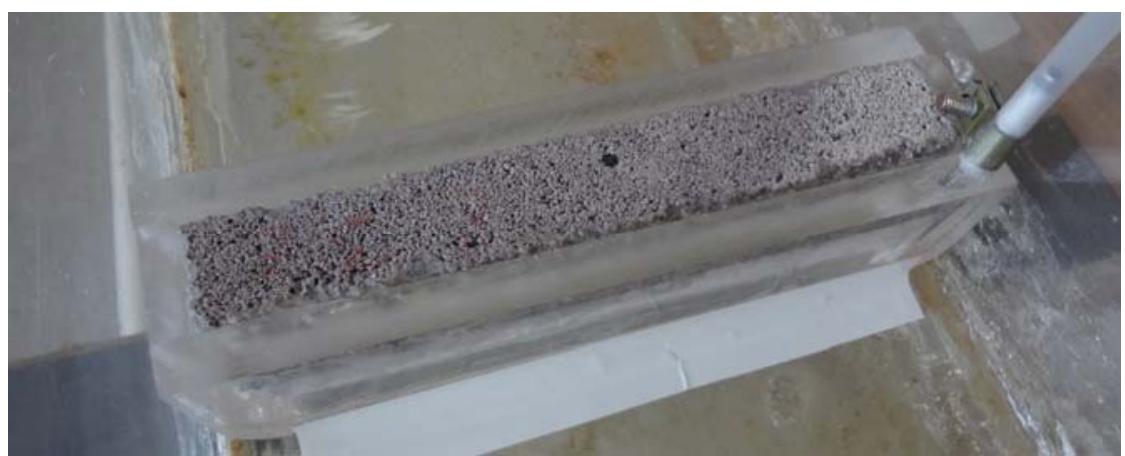

Figura 2. Plugue com elemento poroso de dimensões (20x150x20)mm.

A cortina de gás teve sua origem em elementos porosos de dimensões controladas, alimentados por gás, nesse caso por ar atmosférico comprimido. A vazão de ar comprimido foi controlada por rotâmetro e a pressão da linha por um manômetro.

\subsection{Avaliação da eficiência de remoção de partículas pela aplicação do sensor APS III}

A avaliação da eficiência de remoção das inclusões foi feita pela aplicação da sonda APS III, Aqueous Particle Sensor 3, que faz a contagem on-line de partículas durante o ensaio. Essa sonda é mostrada na Figura 3.

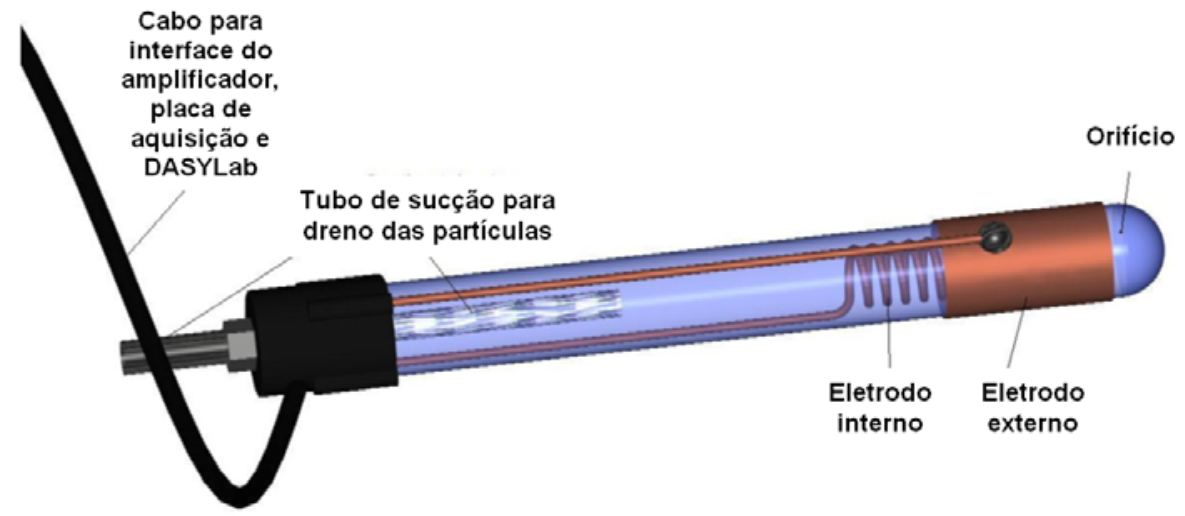

Figura 3. APS III - Aqueous Particle Sensor 3 [9].

Chakraborty [9] apresentou o princípio de funcionamento da forma de detecção de partículas pela Zona de Sensibilidade Elétrica da APS III. Ela se baseia na existência de uma diferença na condutividade elétrica entre a partícula e o fluido de trabalho.

$\mathrm{Na}$ Figura 4 é mostrado o princípio de funcionamento da APS III. O fluido, que contém uma suspensão de partículas simulando inclusão, passa através de um orifício estreito de tamanho conhecido. Dois eletrodos são utilizados para aplicar uma corrente elétrica constante (I) através do orifício. Quando o orifício não contém partículas, a resistência $(R)$ entre os eletrodos é devido apenas ao fluido condutor. Isto produz uma diferença de potencial $V_{0}=(\mathrm{RI})$ através dos eletrodos, que é registrada como a saída de linha de referência para a ausência de partículas. Agora, considere uma única partícula que entra no orifício, ou na Zona de Sensibilidade Elétrica, deslocando, assim, o seu próprio volume de fluido. Uma vez que o fluido condutor no orifício é agora substituída por uma partícula eletricamente isolante, a resistência através do orifício altera para $(R+\Delta R)$ e a diferença de potencial através dos eletrodos salta para $V_{1}=(R+\Delta R) l$. Este salto de tensão, quando a partícula passa através do orifício, é gravado e a sua magnitude é a indicação do tamanho da partícula [10-11]. 


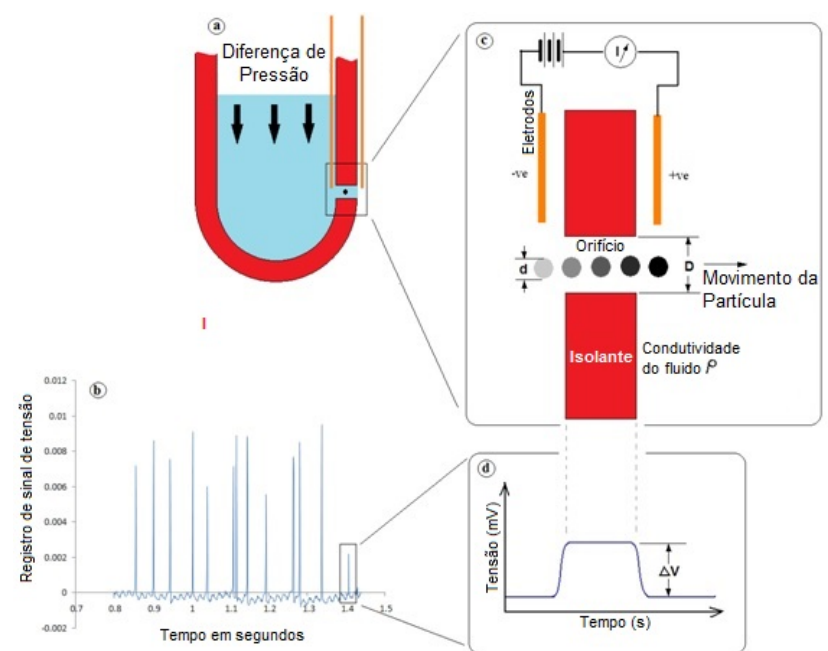

Figura 4. Princípio da Zona de Sensibilidade Elétrica [9].

Cada teste (de posição de plugue com a vazão de gás) foi feito em triplicata com um sensor APS III, gerando três resultados. As condições empregadas foram:

a) Teste de bancada da APS III, para referência da densidade e distribuição de tamanho de partículas no fluxo de entrada do distribuidor (off-line);

b) Sem modificadores de fluxo (barragens e diques) e sem injeção de gás;

c) Sem modificadores de fluxo e com injeção de gás por plugue poroso.

$\mathrm{Na}$ Figura 5 é apresentado esquematicamente cada posicionamento de plugue no modelo físico, e a localização da APS III e dos condutivímetros.

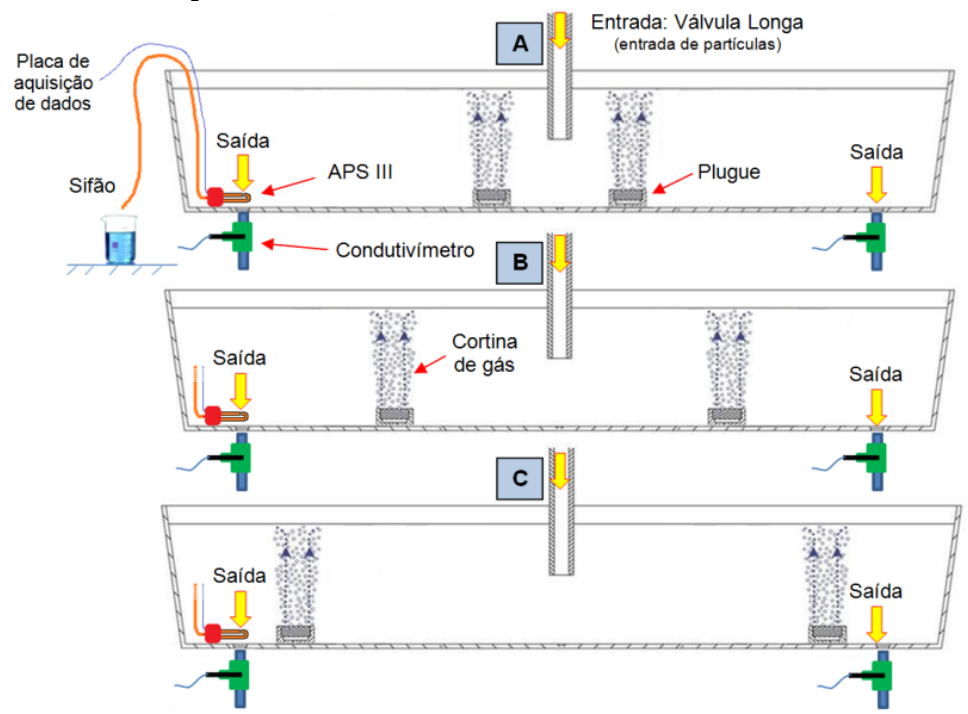

Figura 5. Posicionamento simétrico dos plugues no distribuidor para A: $28 \mathrm{~cm}, \mathrm{~B}: 56 \mathrm{~cm}$ e

C: $84 \mathrm{~cm}$, todos em relação a válvula longa.

Microesferas ocas de borossilicato foram usadas para simular as inclusões, e elas foram injetadas na entrada da válvula longa por uma bomba peristáltica. A poupa de partículas usada tinha a concentração de $1 \mathrm{~g} / \mathrm{l}$ e foi injetada com a vazão de $68 \mathrm{ml} / \mathrm{min}$ no sistema.

Distribuição granulométrica passante de especificação das partículas é: $20 \mu \mathrm{m}(10 \%)$; $38 \mu \mathrm{m}(50 \%) ; 60 \mu \mathrm{m}(90 \%)$ e $68 \mu \mathrm{m}(97 \%)$, e simulam a ação de partículas inclusionárias na escala industrial [12] de 28, 54, 85 e $96 \mu \mathrm{m}$ respectivamente.

Cada ensaio teve duração, aproximadamente, de 6 tempos de residência teóricos [7]. $O$ cálculo de eficiência de remoção de partículas foi feito conforme Chakraborty [9]. 


\subsection{Determinação das Curvas DTR}

Para a realização dos testes relativos à obtenção das curvas DTR, em cada saída de veio foi conectado um sensor de condutividade elétrica. Após estabelecida a altura padrão de água no distribuidor e o escoamento entrar em regime estacionário, foi feita a injeção, na entrada da válvula longa, de um traçador iônico, cloreto de potássio em solução aquosa, na forma de um pulso com duração inferior a 2 segundos. Esse traçador altera a condutividade elétrica da água, fazendo com que os sensores de condutividade detectem essa variação em função do tempo, que mais tarde foi transformada em concentração através de uma curva de calibração. O tratamento dos dados de variação de condutividade em função do tempo permite a determinação da curva DTR. Em geral, o tempo de aquisição de dados durou 4 tempos de residência teóricos do distribuidor. Durante todo o ensaio, os condutivímetros permaneceram conectados a um computador, onde foram registrados os dados experimentais.

Para cada ensaio, foi obtida uma curva DTR específica normalizada em função do tempo e da concentração, ambos adimensionais. Os valores das frações dos volumes característicos (pistonado, morto e mistura) foram extraídos de sua respectiva curva DTR conforme metodologia descrita por Sahai e Emi [7].

As curvas DTR foram levantadas para as mesmas combinações de posição de plugue e vazão de gás injetado utilizadas na avaliação da eficiência de remoção de partículas por meio da APS III.

Cada teste (de posição de plugue com a vazão de gás) foi feito em triplicata com dois sensores de condutividade, gerando seis resultados. As condições empregadas foram:

a) Sem modificadores de fluxo e sem injeção de gás;

b) Sem modificadores de fluxo e com injeção de gás por plugue poroso.

\section{RESULTADOS E DISCUSSÃO}

\subsection{Avaliação da eficiência de remoção de partículas via sonda APS III}

Avaliou-se a eficiência de remoção inclusões para o distribuidor sem modificadores de fluxo. Os ensaios foram feitos com três repetições, para três níveis de posição de plugue: 28,56 e $84 \mathrm{~cm}$ de afastamento da válvula longa, e para quatro níveis de vazão de gás: 1, 2, 3 e 4NI/min. Na Figura 6 é comparada a eficiência de remoção de inclusões entre os ensaios e a referência sem injeção de gás, a partir da média amostral de cada grupo de ensaios com injeção de gás e de seu respectivo intervalo de $95 \%$ de confiança.

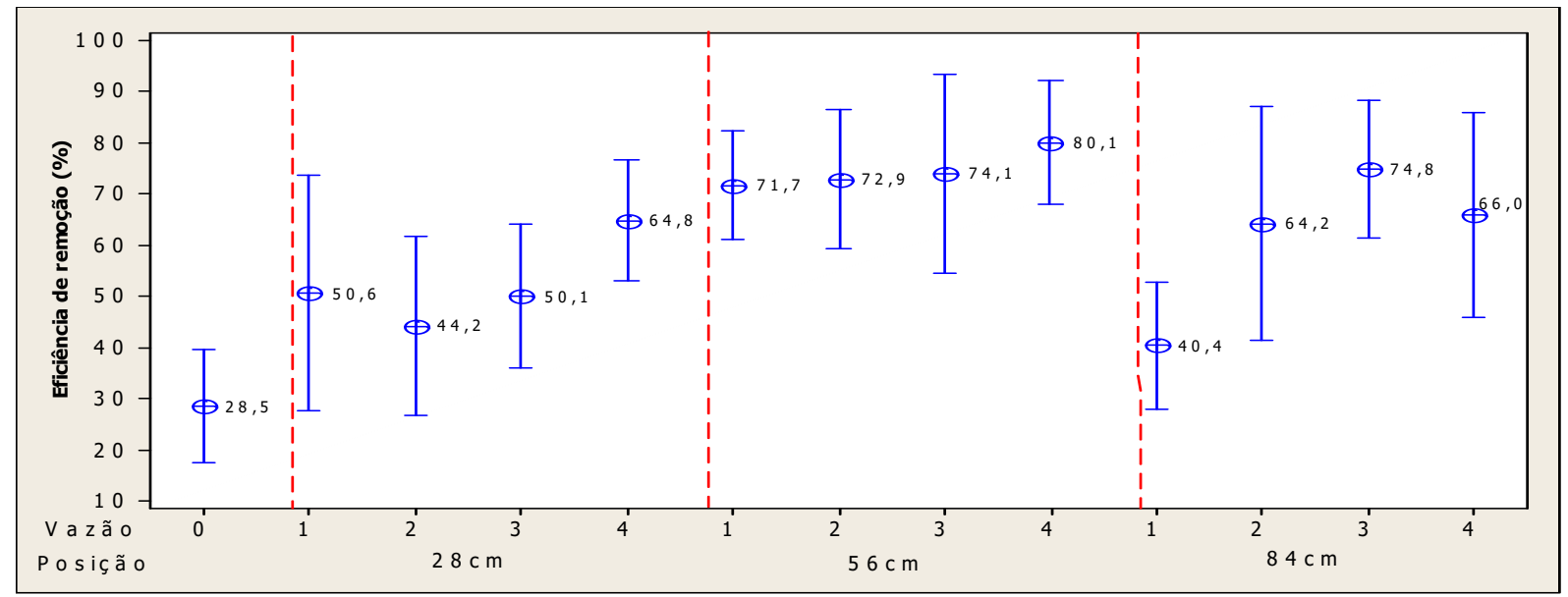

Figura 6. Comparação da eficiência de remoção de partículas do distribuidor sem modificadores de fluxo, sem e com injeção de gás variando a posição e vazão de plugue. 
Na Figura 6 é apresentada evidência estatística, com nível de significância de $5 \%$, de que a vazão de gás injetada afetou a eficiência de remoção de inclusões do distribuidor sem modificadores de fluxo, para as configurações:

- plugue a $28 \mathrm{~cm}$ da válvula longa para a vazão de $4 \mathrm{NI} / \mathrm{min}$;

- plugue a $56 \mathrm{~cm}$ da válvula longa para as vazões de 1, 2, 3 e $4 \mathrm{NI} / \mathrm{min}$;

- plugue a $84 \mathrm{~cm}$ da válvula longa para as vazões de 2,3 e $4 \mathrm{NI} / \mathrm{min}$.

A eficiência de remoção de inclusões para a posição de plugue $28 \mathrm{~cm}$ e vazões de $1,2 \mathrm{e}$ $3 \mathrm{NI} / \mathrm{min}$ apresentou dispersão de resultado. No sistema foi observado uma cortina pouco estável devido à perturbação do escoamento próximo ao jato da válvula longa. A posição de plugue que mais se destacou foi a intermediária, onde todas as vazões de gás injetadas mostram efeitos relevantes. Também se observa para essa posição, pela tendência linear da média amostral, que existe espaço para investigação do aumento da vazão de gás, contudo a energia de agitação da superfície deve ser avaliada, uma vez que existe a possibilidade de reversão de inclusões para o fluido. Outro aspecto é que todos esses resultados não são diferentes entre si pela análise de seus respectivos intervalos de $95 \%$ de confiança. A posição de plugue a $84 \mathrm{~cm}$ sugere, pela tendência das médias, a existência de um máximo de eficiência para a vazão de $3 \mathrm{NI} / \mathrm{min}$.

Para modelar o comportamento geral da eficiência de remoção de inclusões do sistema em estudo, criou-se a Figura 7, com base nos resultados de eficiência de remoção dos dados que deram origem a Figura 6. Ela apresenta um gráfico das curvas de nível da superfície de resposta com linhas de isoeficiências de remoção de partículas, em função dos fatores vazão de gás e posição de plugue.

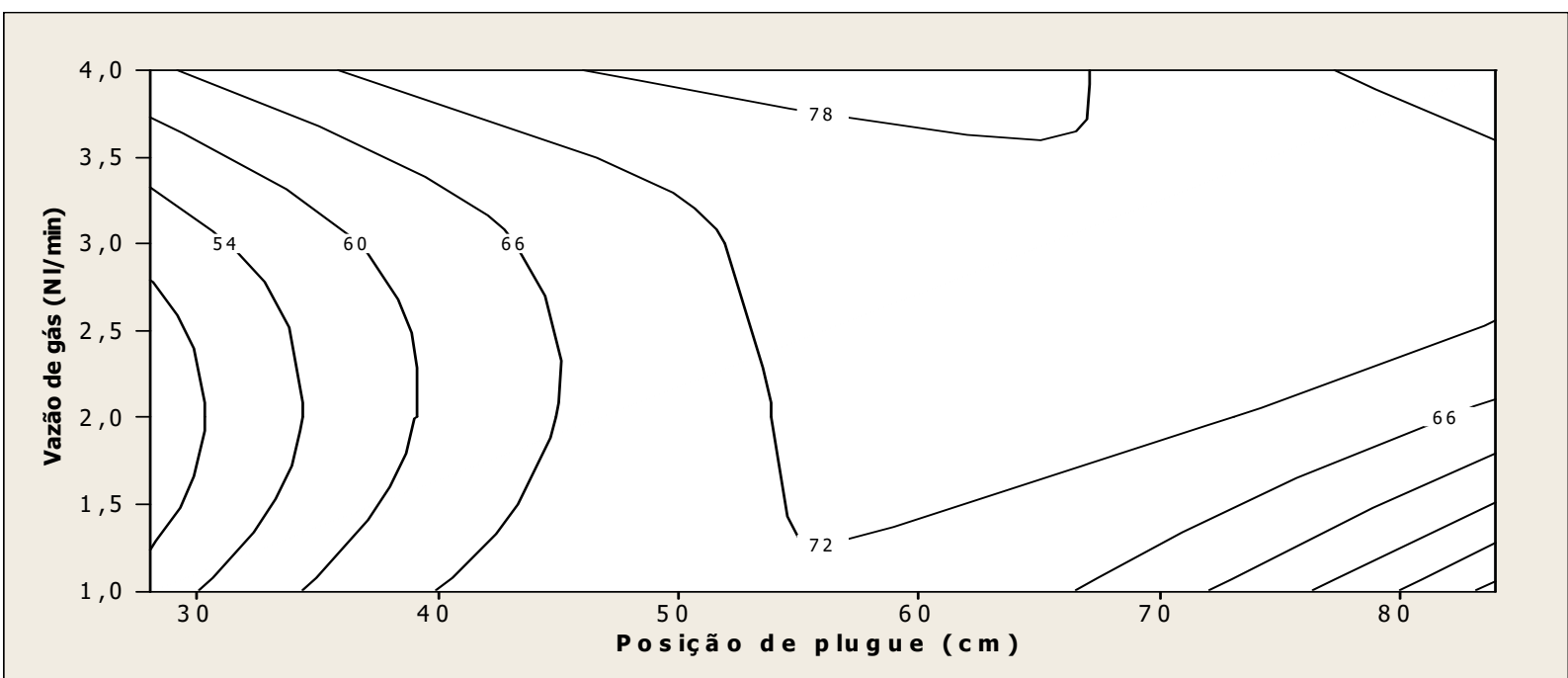

Figura 7. Curvas de nível da superfície de resposta para a eficiência de remoção de partículas para distribuidor sem modificadores de fluxo, e com injeção de gás de 1 a $4 \mathrm{NI} / \mathrm{min}$ para as posições variando entre 28 a $84 \mathrm{~cm}$ de afastamento da válvula longa.

Na Figura 7 é revelada a existência de uma região central onde a eficiência supera $72 \%$ de remoção, iniciando a aproximadamente $57 \mathrm{~cm}$ da válvula longa e para uma vazão de $1,3 \mathrm{NI} / \mathrm{min}$. Contudo, a maior eficiência de remoção encontra-se na ordem de $78 \%$ a aproximadamente $64 \mathrm{~cm}$ da válvula longa para uma vazão de $3,8 \mathrm{NI} / \mathrm{min}$. Essa última vazão poderá gerar um alto nível de agitação na superfície de banho e tem potencial para reverter inclusões não metálicas que não foram absorvidas pela escória, ou até mesmo incorporar a escória ao banho por arraste.

Para o refinamento da análise, foi empregada a técnica da análise de variância (ANOVA) para a eficiência de remoção de partículas. A Figura 8, referente à ANOVA, foi obtida do software Minitab16. 


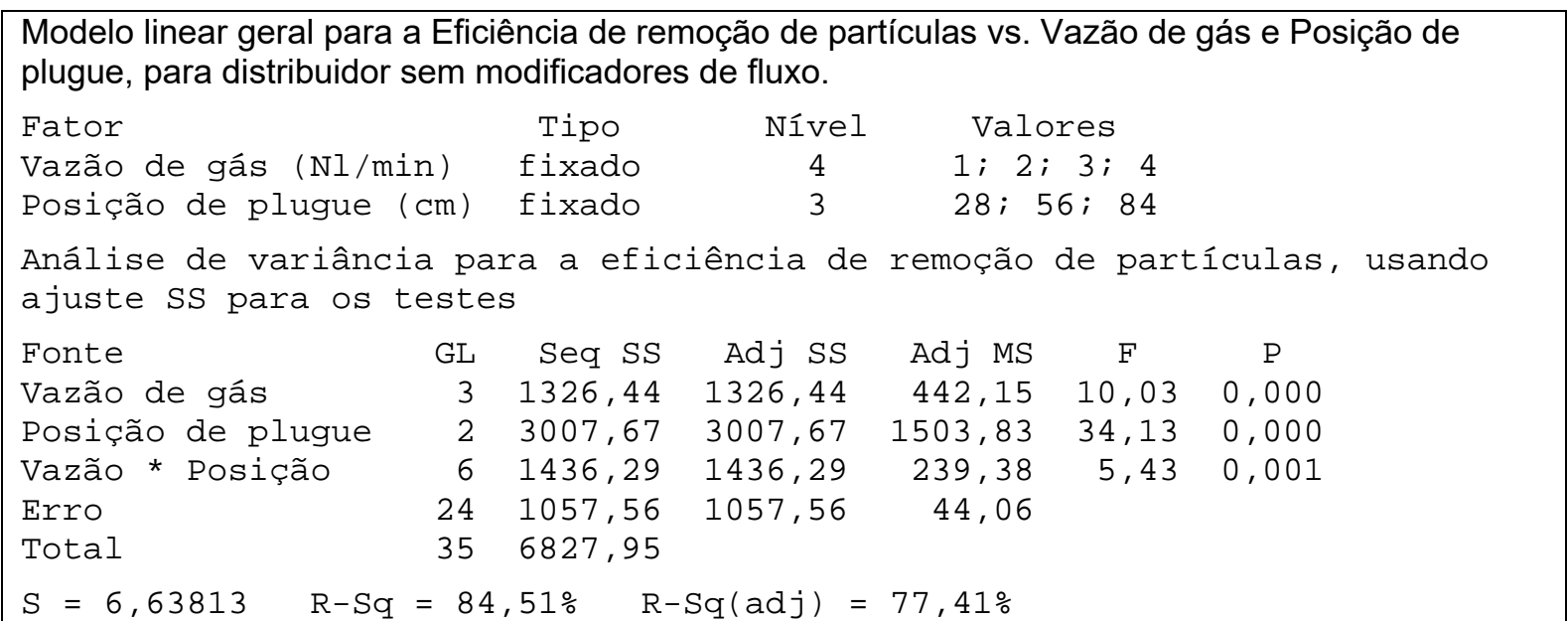

Figura 8. Análise de variância para a eficiência de remoção de partículas para as configurações do distribuidor sem modificadores de fluxo, e com injeção de gás de 1, 2, 3 e 4NI/min para as posições a 28,56 e $84 \mathrm{~cm}$ da válvula longa.

Pela análise da ANOVA, apresentada na Figura 8, conclui-se que todos os fatores, e sua combinação, têm efeito significativo sobre a variável resposta, eficiência de remoção, haja vista que, para um nível de significância de $5 \%$, todos os p-valores se apresentaram menores que esse valor de referência e, portanto, estão na região de rejeição da hipótese nula de que os efeitos não são significativos. Na última linha da ANOVA pode-se ver o desvio padrão amostral (S), o coeficiente de determinação $R^{2}$ e o coeficiente de determinação ajustado $R^{2}$ (adj). O coeficiente de determinação $R^{2}$ indica que $84,51 \%$ do resultado aqui observado da eficiência de remoção pode ser explicado pelos fatores estudados. A fração complementar de $\mathrm{R}^{2}$ faz referência aos ruídos do sistema, ou seja, variáveis não controladas, mas também é válido salientar que a própria turbulência do escoamento introduz alguma variabilidade no sistema, de forma que o $\mathrm{R}^{2}$ nunca será 1 , mesmo com todas as variáveis externas (temperatura, condutividade da água, ruídos elétricos, etc.) controladas. Por fim, o coeficiente de determinação ajustado corrige o coeficiente de determinação original levando em consideração o tamanho da amostra.

Na Figura 9 é apresentada a análise dos efeitos principais na eficiência de remoção de partículas para as configurações do distribuidor sem modificadores de fluxo estudadas.

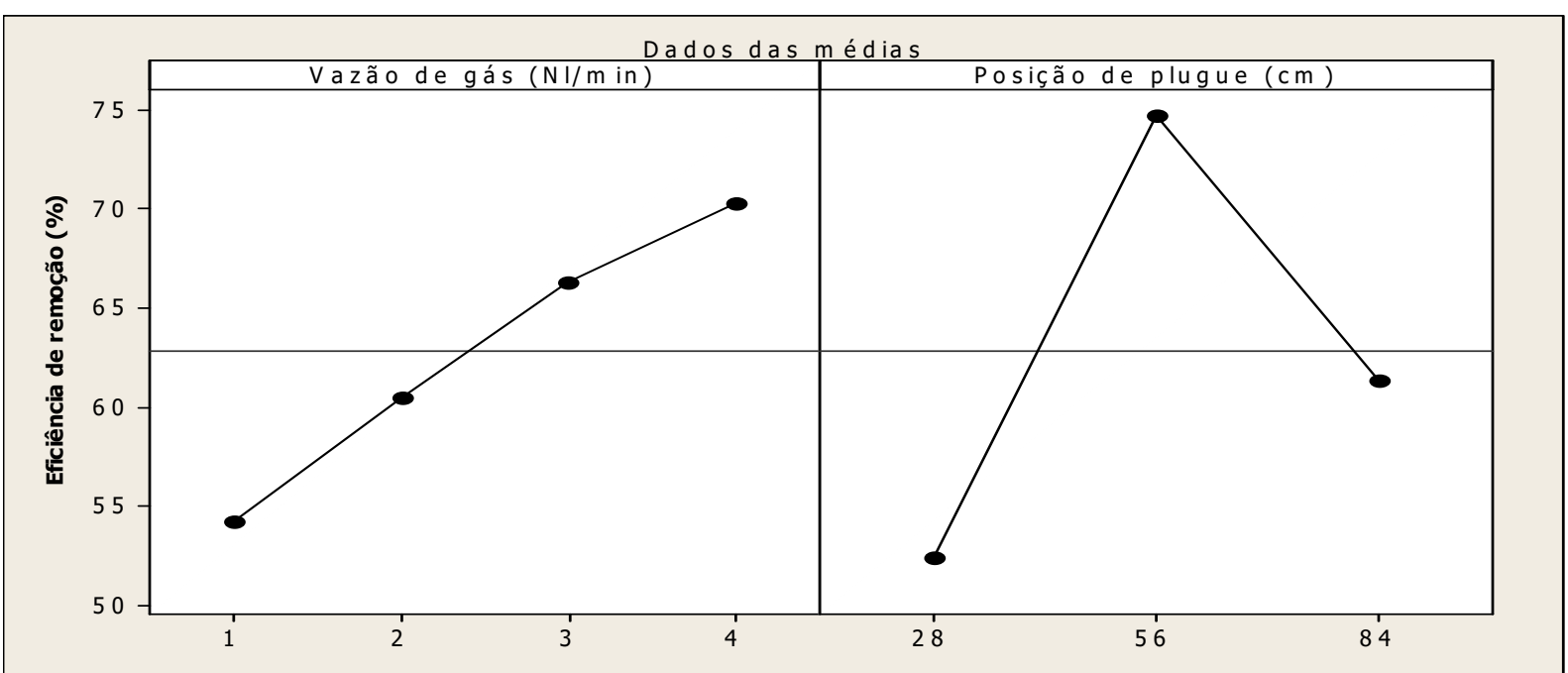

Figura 9. Análise dos efeitos principais na eficiência de remoção de partículas para as configurações do distribuidor sem modificadores de fluxo e com injeção de gás de 1, 2, 3 e 4NI/min para as posições a 28,56 e $84 \mathrm{~cm}$ da válvula longa. 
Na Figura 9 são expostos os dois fatores desse sistema em estudo: vazão de gás e posição de plugue. Com relação à vazão, observa-se que a eficiência de remoção aumenta com a elevação da vazão de forma aproximadamente linear. Já em relação à posição do plugue, observa-se que existe um comportamento não linear, o que sugere a existência de uma distância ótima para a posição de plugue. Essa afirmação torna-se mais notável uma vez que a ANOVA da Figura 8 apresentou o maior valor de $\mathrm{F}$ (relação entre sinal e ruído) para o fator posição. Pela análise das intensidades, o fator posição do plugue foi o que mais contribuiu favoravelmente para a eficiência de remoção de partículas, e em particular, o posicionamento do plugue a $56 \mathrm{~cm}$ da válvula longa foi o mais relevante e tem maior poder de explicação estatística. Outro aspecto relevante é a maior faixa (range) de variação de eficiência vista entre as posições 28 e $56 \mathrm{~cm}$ (que também se traduz nos maiores afastamentos médios da média global), que conduz a inclinação mais acentuada do fator posição.

\subsection{Volumes característicos e eficiência de remoção de inclusões}

A razão entre o volume pistonado e o volume morto é um índice para o estudo da remoção de inclusões a partir do ensaio de distribuição de tempo de residência [13]. Como o volume pistonado auxilia no direcionamento das inclusões à escória em escoamentos preferencialmente ascendentes e o volume morto diminui o tempo de residência do aço (prejudicial à flotação das inclusões), deseja-se que o primeiro aumente o máximo possível e o último diminua. Logo, quanto maior for a razão entre esses volumes, melhor seriam as condições de flotação de inclusões.

Tabela 1. Volumes característicos, razão entre volume pistonado e volume morto, e eficiência de remoção para o distribuidor sem modificadores de fluxo, sem e com injeção de gás de 1, 2, 3 e 4NI/min, para as posições de plugue a 28, 56 e $84 \mathrm{~cm}$ da válvula longa

\begin{tabular}{|c|c|c|c|c|c|c|c|}
\hline \multirow{2}{*}{$\begin{array}{c}\text { Tratamento } \\
\text { (n) }\end{array}$} & \multirow{2}{*}{$\begin{array}{c}\text { Posição } \\
\text { (cm) }\end{array}$} & \multirow{2}{*}{$\begin{array}{l}\text { Vazão } \\
\text { (NI/min) }\end{array}$} & \multicolumn{3}{|c|}{ Volumes (\%) } & \multirow{2}{*}{$\begin{array}{l}\text { Razão } \\
\text { Vp/Vm }\end{array}$} & \multirow{2}{*}{$\begin{array}{c}\text { Eficiência } \\
\text { remoção } \\
(\%)\end{array}$} \\
\hline & & & $\begin{array}{c}\text { Pistonado } \\
\text { Vp } \\
\end{array}$ & $\begin{array}{c}\text { Morto } \\
\text { Vm }\end{array}$ & $\begin{array}{c}\text { Mistura } \\
\text { Vmist }\end{array}$ & & \\
\hline 1 & - & 0 & 22,36 & 16,71 & 60,93 & 1,35 & 28,5 \\
\hline 2 & 28 & 1 & 18,48 & 16,43 & 65,10 & 1,14 & 50,6 \\
\hline 3 & 28 & 2 & 15,81 & 18,81 & 65,38 & 0,84 & 44,2 \\
\hline 4 & 28 & 3 & 14,22 & 20,90 & 64,88 & 0,68 & 50,1 \\
\hline 5 & 28 & 4 & 15,82 & 21,63 & 62,56 & 0,73 & 64,8 \\
\hline 6 & 56 & 1 & 19,68 & 17,75 & 62,57 & 1,11 & 71,7 \\
\hline 7 & 56 & 2 & 13,83 & 19,54 & 66,63 & 0,71 & 72,9 \\
\hline 8 & 56 & 3 & 14,18 & 20,13 & 65,69 & 0,71 & 74,1 \\
\hline 9 & 56 & 4 & 12,79 & 21,19 & 66,03 & 0,61 & 80,1 \\
\hline 10 & 84 & 1 & 18,35 & 22,19 & 59,46 & 0,83 & 40,4 \\
\hline 11 & 84 & 2 & 14,81 & 26,37 & 58,82 & 0,56 & 64,2 \\
\hline 12 & 84 & 3 & 16,44 & 24,21 & 59,36 & 0,69 & 74,8 \\
\hline 13 & 84 & 4 & 14,43 & 23,12 & 62,46 & 0,62 & 66,0 \\
\hline
\end{tabular}

Na Figura 10 é exibida a correlação entre a eficiência de remoção de partículas e a razão entre o volume pistonado e volume morto. 


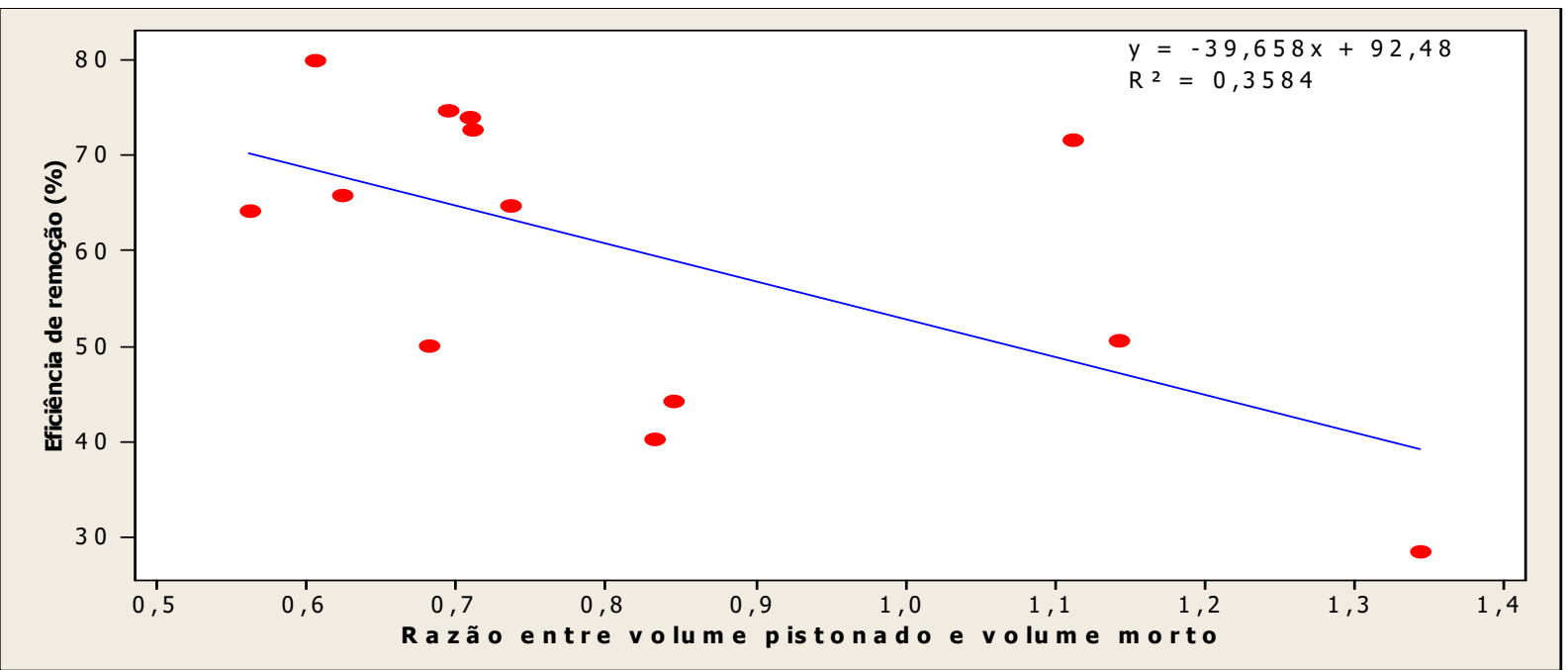

Figura 10. Correlação entre a eficiência de remoção de partículas e a razão entre volume pistonado e volume morto para as vazões de $0,1,2,3$ e $4 \mathrm{NI} / \mathrm{min}$ e posição de plugue a 28,56 e $84 \mathrm{~cm}$ da válvula longa do distribuidor sem modificadores de fluxo.

Esperava-se um coeficiente de inclinação positivo para a reta estabelecida entre as duas variáveis, entretanto na Figura 10 é exibido um comportamento inverso entre as variáveis. Assim, para a configuração estudada, apenas a análise pura dos volumes característicos desse distribuidor não é adequada para indicar a melhor configuração interna objetivando a máxima eficiência de remoção de inclusões. Pereira [14], em sua tese de doutorado, também fez essa mesma constatação, onde afirmou que associar um maior índice de flotação de inclusões a uma maior fração de volume em escoamento pistonado com uma menor fração de volume morto não é sempre válida. Outro aspecto sobre a análise das curvas características é o fato dessa metodologia não levar em consideração a remoção de inclusões pelo efeito das bolhas da cortina de gás [13].

\section{CONCLUSÃO}

Pelo presente trabalho é possível concluir que:

- O uso de injeção de gás apresenta ganho significativo na eficiência de remoção de inclusões em relação ao distribuidor sem injeção de gás;

- A melhor eficiência de remoção de inclusões para o distribuidor sem modificadores de fluxo, considerando um nível aceitável de agitação na superfície do banho, foi para a configuração com plugues posicionados a $56 \mathrm{~cm}$ de afastamento da válvula longa e operando com uma vazão de $1,3 \mathrm{NI} / \mathrm{min}$;

- A análise pura dos volumes característicos do distribuidor não é adequada para indicar a melhor configuração interna objetivando a máxima eficiência de remoção de inclusões.

\section{Agradecimentos}

Os autores agradecem à FAPEMIG, pelo apoio financeiro concedido ao projeto de número de processo TEC-PPM-0118-13, intitulado: "Modelagens Física e Matemática do Escoamento Multifásico em Reatores Metalúrgicos";

Os autores agradecem também o apoio da à CAPES/PROEX ao programa de pósgraduação PPGEM/UFMG;

Os autores agradecem aos estudantes de Engenharia Metalúrgica: Filipe Alvarenga Araújo, Laura Oliveira Lamas, Lorena Campolina Milan Lucrécio e Pedro Henrique da Rocha Silva pelas contribuições técnicas para a realização deste trabalho. 


\section{REFERÊNCIAS}

1 Sinha AK, Sahai Y. Mathematical modeling of inclusion continuous casting tundishes transport and removal. ISIJ International. 1993;33(5):556-566.

2 Nascimento RP. Remoção de inclusões não-metálicas do aço líquido através do borbulhamento de argônio pelo fundo do distribuidor de lingotamento contínuo da ArcelorMittal Tubarão [Dissertação, Mestrado]. Ouro Preto: Escola de Engenharia da UFOP; 2008. p. 17; 55-69.

3 Morales RD, Banderas AR, Demedices LG, Cruz MD. Mathematical simulation and modeling of steel flow with gas bubbling in trough type tundishes. ISIJ International. 2003;43(5):653-662.

4 Rogler JP. Modeling of inclusion removal in a tundish by gas bubbling [Dissertação de Mestrado]. Toronto: Ryerson University; 2004. p. 57; 65.

5 Seshadri V, Tavares RP, Silva CA, Silva IA. A physical modelling study of inclusion removal in tundish using inert gas curtain. Tecnologia em Metalurgia, Materiais e Mineração. 2012;9:22-29.

6 Zhang MJ, Gu HZ, Huang A, Zhu HX, Deng CJ. Physical and mathematical modeling of inclusion removal with gas bottom-blowing in continuos casting tundish. Journal of Mining and Metallurgy. 2011;47:37-44.

7 Sahai Y, Emi T. Melt flow characterization in continuous casting tundishes. ISIJ International.1996;36(6):667-672.

8 Wollmann AM. Estudo de fluxo em um distribuidor de lingotamento contínuo [Dissertação de Mestrado]. Porto Alegre: Escola de Engenharia da UFRGS; 1999. p. 8-29.

9 Chakraborty A. Development of an on-line aqueous particle sensor to study the performance of inclusions in a 12 tonne, delta shaped full scale water model tundish [Dissertação de Mestrado]. Montreal: McGill University; 2010.

10 Guthrie RIL, Li M. In situ detection of inclusions in liquid metals: Part I. Mathematical modeling of the behavior of particles traversing the electric sensing zone. Metallurgical and Materials Transactions B. Dec 2001;32B:1067.

11 Guthrie RIL, Li M. In situ detection of inclusions in liquid metals: Part II. Metallurgical applications of LiMCA systems. Metallurgical and Materials Transactions B. Dec 2001;32B:1081.

12 Sahai Y, Emi T. Criteria for water modeling of melt flow and inclusion removal in continuous casting tundishes. ISIJ International. 1996;36(9):1166-1173.

13 Machado FD. Modelagem física de remoção de inclusões em distribuidor de lingotamento contínuo de tarugos [Dissertação de Mestrado]. Porto Alegre: Escola de Engenharia da UFRGS; 2014. p. 21-36.

14 Pereira ROS. Modelamento matemático do escoamento turbulento, da transferência de calor e da solidificação no distribuidor e na máquina de lingotamento contínuo [Tese de Doutorado]. Belo Horizonte: Escola de Engenharia da UFMG; 2004. p. 220. 